\title{
Asteroid watchers debate false alarm
}

[WASHINGTON] A worldwide media frenzy last week about reports of a possible asteroid collision with the Earth in 2028 has led to scientists taking a close look at their strategy for informing the public about such events.

Many experts in the field are critical of a decision by the Central Bureau for Astronomical Telegrams in Cambridge, Massachusetts, to send out a press release warning of a possible collision before first checking with other researchers. But others say the false alarm was an inevitable by-product of the open way in which asteroid researchers share information.

The furore began on 11 March, when the bureau - a non-profit organization that serves as a clearing-house for astronomical alerts - e-mailed a 'circular' predicting that the mile-wide asteroid 1997 XF11 would pass very close to Earth in 2028, on the basis of observations made a week earlier. The circulars go out to subscribing astronomers, but some journalists also receive them.

Anticipating that the story would spread quickly, Stephen Maran, an astronomer at the US space agency NASA's Goddard Space Flight Center in Maryland, and long-time press officer for the American Astronomical Society (AAS), suggested to the bureau's director, Brian Marsden, that he send a more general release through the AAS to science reporters around the world.

Marsden, an expert on asteroid orbits, then issued a release including the statement: "The chance of an actual collision is small, but one is not entirely out of the question." The news of a potentially devastating asteroid impact made headlines around the world.

Ted Bowell of the Lowell Observatory in Arizona, whose search for near-Earth objects

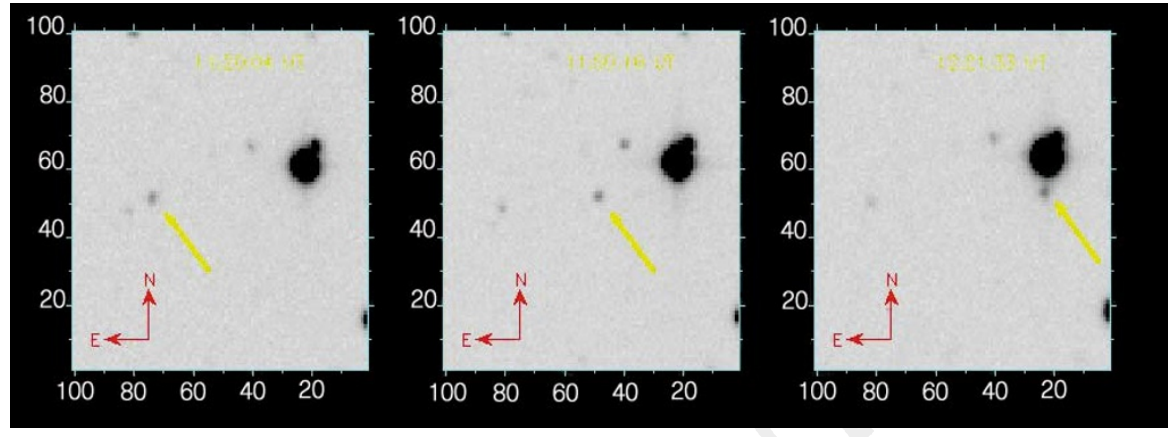

Watch out: the first images of 1997 XF11 from Kitt Peak's Spacewatch telescope, 6 December 1997.

will soon join two other NASA-funded searches already under way, is among those who believe the warning was released prematurely. Marsden, he says, "should have consulted with several of his colleagues before rushing into print".

Eleanor Helin of NASA's Jet Propulsion Laboratory, who runs a search called NEAT, and Bowell turned up photographs of the asteroid from 1990 in less than a day, allowing more accurate orbit measurements. The new data placed the object a safe 600,000 miles from Earth at its closest approach.

"I don't think we were crying wolf," says Marsden, pointing out that the small community of asteroid researchers usually works this way, sharing orbital data and then asking colleagues to make new observations or search for archival data to improve the estimates. He also says that until Helin's photos surfaced, three of four groups calculating the asteroid's orbit agreed that there was a remote but 'non-zero' chance of a collision in 2028.

Helin agrees that the system of communication by circular generally works, and says "we couldn't have acted any faster".

\section{Decline in Indian publications raises alarm}

[NEW DELHI] A steady decline in the international profile of Indian science has been revealed by an analysis of research papers published in recent years. This "is a cause of concern", says the study.

India has fallen from eighth largest publishing nation in 1989 to twelfth position, with just a 2 per cent share of the world's journal articles — a significant drop from the 2.8 per cent in 1989 .

The study was carried out by Subbiah Arunachalam of the Indian Institute of Technology in Chennai and R. Srinivasan and Vidyalakshmi Raman of the Central Electrochemical Research Institute in Karaikudi, and the results are presented in the latest issue of Current Science, published by the Indian Academy of Sciences.

The authors analysed papers originating in India and published in 2,300 journals indexed in the Science Citation Index (SCI) in 1989-92. Nearly a quarter of the papers from India are in chemistry, 18 per cent in physics, and 13 per cent in engineering. Biology accounted for 12 per cent.

Although India's share of the world's journal articles decreased marginally, there has been a significant increase in the number of papers published by Indian scientists in foreign journals as a whole, rising from 8,751 in 1989 to 9,958 in 1992 . In contrast there was a 27 per cent decline in the number of papers published in the 12 Indian journals indexed in SCI.

India invested US\$1,460 million -0.83 per cent of its gross national product - in research and development in science and technology in 1992-93, a figure that has been steadily declining from 0.93 per cent in 1989-90.
David Morrison of the NASA Ames Research Center, who led a comprehensive assessment in 1992 of the asteroid threat, agrees "it would have been smarter for the community to seek those [other archived observations] before going public". But, he says, "I'd much rather that we had an open system, even if it might sometimes embarrass us, than that somebody try to control it."

Scientists involved in the Search for Extraterrestrial Intelligence (SETI) have addressed the problem by agreeing to a protocol whereby anyone who detects a suspected extraterrestrial signal would consult privately with other colleagues before going public with the news. This may not work, however, in the asteroid community, which has longlasting rivalries. "Maybe the SETI people are more harmonious," jokes Marsden.

The flap about 1997 XF11 was expected to be a hot topic among asteroid researchers meeting this week in Houston, Texas, to discuss how the various campaigns to identify new asteroids are faring, and how the search might be accelerated.

The meeting, sponsored by NASA, had been planned before last week's events. Morrison's 1992 report called for spending approximately $\$ 5$ million a year for 10 years to survey the population of Earth-crossing objects more than one kilometre in diameter, 90 per cent of which have not yet been identified.

But NASA is currently spending only $\$ 1$ million a year. "At the current rate of discovery, it will take more than a century to find 90 per cent or more of the objects [the size of 1997 XF11] with Earth-crossing orbits," says Morrison.

Congress may call for stepping up the pace given the worldwide attention focused last week on the asteroid threat. The day after Marsden's announcement, the chairman of the House of Representatives space subcommittee, Dana Rohrabacher (Republican, California), called on President Bill Clinton to reverse his veto of the Pentagon-sponsored project Clementine 2, which would fire small interceptors into the surface of an asteroid (see Nature 389, 776; 1997). Tony Reichhardt 